\title{
Size-frequency distribution of earthquakes in hierarchically organized load-transfer models
}

\author{
J. B. Gómez ${ }^{1}$ and A. F. Pacheco ${ }^{2}$ \\ 'Departamento de Ciencias de la Tierra, Universidad de Zaragoza, 50009 Zaragoza, Spain \\ ${ }^{2}$ Departamento de Física Teórica, Universidad de Zaragoza, 50009 Zaragoza, Spain
}

Received: 8 December 1997 - Accepted: 3 July 1998

\begin{abstract}
Large hierarchically organized sets of elements (simulating asperities in a fault) are loaded to the point of complete failure. The fracture thresholds of individual elements are stochastically distributed, and the hierarchical structure for load-transfer is of the fractaltree type. During the breakdown process there occur bursts (earthquakes) of several elements breaking simultaneously at a given load. Using Monte Carlo simulations we compute the frequency of bursts versus their size. This shows a gross power-law behaviour superimposed by a wavy pattern closely related to the coordination number of the fractal tree used for the load-transfer structure.
\end{abstract}

\section{Introduction}

The statistical properties of the strength of materials with stochastically distributed elements are important in applied science. A simple classical group of models in this area falls within the fiber-bundle paradigm which has been studied by many authors in both the static and time-dependent versions (Coleman, 1957; Daniels, 1945; Newman et al., 1994; Phoenix, 1978; Sornette, 1989, etc). In the static version, one of the properties most intensively studied is the strength of the set, i.e., the average value of stress (or load) at which a system composed of $N$ elements fails. Another interesting property is the size-frequency relation of the breaking bursts as - one progressively increases the stress until complete failure. In this paper, the analysis of this second property in relation to an important type of fiber-bundle model will be our objective.

Fiber-bundle models can be classified according to the way load supported by failed fibers is transferred to the surviving ones. In the simplest case, the set obeys the equal load-sharing (ELS) rule; i.e., the non-failed fibers

Correspondence to: J.B. Gómez share the total load equally, and all failed fibers carry no load (Daniels, 1945). This idea can be generalized to formulate local load-sharing (LLS) (Phoenix, 1978) and hierarchical load-sharing (HLS) (Turcotte et al., 1985) models.

In the static case, for the ELS model, Hemmer and Hansen (1992) proved that the expected number $D(n)$ of bursts of size $n$ (in which a number $n$ of elements break simultaneously) follows, for $N \rightarrow \infty$, a universal power law

$D(n) \propto n^{-5 / 2}$.

On the other hand, the universally observed Gutenberg-Richter relation for regional seismicity (Gutenberg and Richter, 1944)

$\log _{10} N=a-b M_{l}$,

with $N$ being the cumulative number of earthquakes with magnitude greater than $M_{l}$ and $a$ and $b$ constants $(.8<b<1.1)$, is of the Eq. 1 type power-law when $M_{l}$ is expressed in terms of energy $E$ (seismic moment); $\log _{10} E=c+d M_{l}(c \approx 11, d \approx 1)$ (Kanamori and Boschi, 1983). Thus, copious research has been done to explore whether other versions of fiber-bundle models also verify this type of power-law size-frequency distribution, as the ELS model does. [It is interesting to note that the ELS model was applied in seismology before Eq. 1 was known: Jones and Molnar (1979), Kanamori (1981), Lomnitz-Adler (1985)]. In particular, models in which the load transfer is assumed to be local, with and without stress dissipation, have been analysed and approximate power-laws also emerge (Christensen and Olami, 1992; Gómez et al., 1994; Zhang and Ding, 1994). As is obvious, the ultimate goal of these efforts is to gauge the real value of these fiber-bundle models to describe earthquake phenomenology.

An important type of fiber-bundle models originally intended for seismological use is the hierarchical fractaltree type model. This was introduced by Turcotte and 


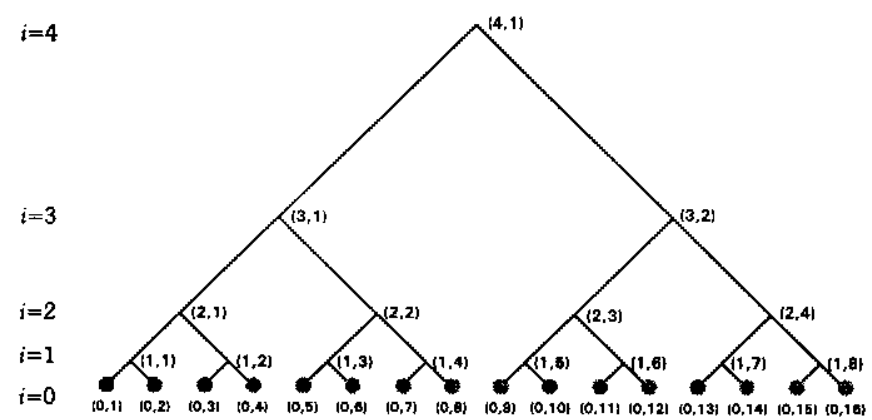

Fig. 1. Fractal tree with coordination number $z=2$ and $k=4$ levels.

collaborators with the aim of describing the stick-slip behaviour of tectonic faults (Smalley et al., 1985; Turcotte et al., 1985). The asperities on a fault were treated as individual elements (equivalent to fibers). In its simplest version, if one element failed, the stress was transferred to the adjacent element on which an induced failure could occur; if two elements failed, the stress was transferred to two adjacent elements, etc. For details see Smalley et al. (1985). This type of organization can also simulate a cable with a hierarchical distribution of components. It is a rigorous result (Newman and Gabrielov, 1991) that in these hierarchical sets the strength $\sigma_{N}$ of a system of $N$ elements decays for large $N$ in the form

$\sigma_{N} \propto[1 / \log \log (N)]$.

As mentioned above, the purpose of this paper is to find out the size-frequency distribution of bursts in this type of hierarchically organized static model. As a fractal-tree structure of transfer is not at all suited for performing Monte Carlo simulations, the computational strategy is detailed in Section 2, together with the emerging results. Finally, in Section 3, we state our conclusions.

\section{Computational Strategy and Results}

The best way of visualizing the hierarchical load transfer scheme is by means of a fractal tree of coordination $z$ and number ol levels $k$. Figure 1 depicts a particular case with $z=2$ and $k=4$.

In the tree, all level-0 elements (filled circles in Fig. 1) and all level $>0$ junctions are numbered level by level from left to right. Level-0 elements are called asperities and level $>0$ elements are called local apexes. Local apexes are the points from which the load distribution operations are performed. A local apex is singled out by a pair of coordinates, the first referring to the level in the hierarchy, and the second to the order of the apex in each level; so, local apex $(2,1)$ is the furthest left apex on the $i=2$ level. As already stated, elements with a zero first coordinate stand for asperities.

In dealing with the elements (local apexes and asperities) of a fractal tree and their relationships, several terms must be defined in advance: the parent of an $l$ level element is this element in level $l+1$ directly joined to it by a trce branch. The neighborhood of an element is the set of $z-1$ elements with a common parent. The range of an element is the set of elements potentially affected by a load transfer event from that element; in particular, the $z$ elements just below an element in its range are its daughters, and the $z^{l}$ level-0 elements in its range are its spanned asperities. For a generic element $(l, m)$ in level $l$, the parent is element $(l+1,\lceil m / z\rceil)$, the neigborhood, plus the element $(l, m)$ itself, is the set $\{(l, j): j=z\lceil m / z\rceil, z\lceil m / z\rceil-1, \ldots, z\lceil m / z\rceil-(z-1)\}$, and the range is the set $\{(l-i, j): i=1,2, \ldots, l, j=$ $\left.(m z)^{i},(m z)^{i}-1, \ldots,(m z)^{i}-\left(z^{i}-1\right)\right\}$. In this last expression, the case $i=1$ gives the daughters of element $(l, m)$, and the case $i=l$ its spanned asperities. In these expressions, $\lceil x\rceil$ means the smallest integer greater than or equal to $x$.

The tree is stored in the computer as a 2-dimensional list of integers with $k$ rows of lengths $z^{k-i},(i=0,1, \ldots$, $k$ ). Level 0 contains $z^{k}(\equiv N)$ asperities, level $1 z^{k-1}$ local apexes, and so on up to level $l$ which contains just one element, the global apex of the fractal tree. When all the spanned asperities of local apex $(l, m)$ have failed we say that the local apex is broken and the $2 \mathrm{D}$ list stores a negative number in position $(l, m)$; if at least one of the spanned asperities of local apex $(l, m)$ is intact we say that the local apex is also intact and the $2 \mathrm{D}$ list stores a positive integer in position $(l, m)$. The total amount of space needed to store the tree structure is $z^{k+1}-1$ integers plus $2 N$ floating-point numbers in the form of two $N$-vectors to store the asperity strengths and loads.

The Monte Carlo simulation of the breaking process can be conveniently summarized in the following steps:

1. Select $z, k$, and the number of realizations to be performed.

2. Select a probability distribution for the strength of the asperities. We have used in all our simulations a Weibull distribution

$p\left(\sigma / \sigma_{0}\right)=1-e^{\left(\sigma / \sigma_{0}\right)^{p}}$,

where $\sigma_{0}$ is a reference load taken here as unity, and $\rho$ is the Weibull index or shape parameter.

3. Assign strengths stochastically to the $N$ asperities according to the selected probability distribution.

4. Raise global load until the breaking point of the asperity satisfying $\min$ (strength - load) $>0$, bearing in mind that already broken asperities have their strengths reduced to zero.

5. Transfer loads consistent with the hierarchical structure until no more asperities break under the applied global load. 
6. Count the number of induced asperity failures in the previous step: by definition this is the size of the $n$th burst.

7. Repeat steps 4-6 until total failure of the tree.

8. Repeat steps 3-7 until the total number of realizations are done.

\section{Compute statistics $D(n)$ versus $n$.}

The only non-trivial step in the above Monte Carlo procedure is step 5, where the transfer of loads among unbroken asperities consistent with the tree structure is made. The main problem is one of book-keeping, and can be reduced to two basic operations: (i) locate the local apex for load distribution in response to the failure of an asperity; ( $i i)$ determine from the local apex of load distribution the set of asperities affected by the load distribution event.

The first operation is carried out by an upward search of the tree until the point (or points) of load redistribution is (are) located. Asperity $m$ (with co-ordinates $(0, m))$ has a hierarchy of local apexes joining it to the global apex; these local apexes have co-ordinates ( $i$, $\left.\left\lceil m / z^{i}\right\rceil\right),(i=1, \ldots k)$. The upward search ends when at least one of the neighbours of a local apex in the hierarchy of asperity $m$ stores a positive value; in this case, the load transferred upwards is shared equally among the intact neighbours, which become the points of load re-distribution for the next basic operation.

During this second operation, the load $\sigma$ assigned to a local apex (and ultimately derived from a previously failed asperity) is re-distributed among the intact local apexes in its range until level- 0 is reached; on each level, the load is shared equally among the intact neighbours. If $\sigma$ is the base load at the point of load distribution in level $l$, the load on each intact local apex in level $l-1$ is $\sigma / z^{\prime}$, where $z^{\prime}$ is the number of intact daugthers; this process of load re-distribution is iterated downwards to level 0 . There, each unbroken asperity receives a part of the initial base load $\sigma$, which is added to the load it was already supporting; if the total new load is greater than the strength of the asperity, the asperity fails and is temporarily stored in a list of failed asperities to be processed; if, on the contrary, the asperity survives the extra load, the only operation to be carried out is the actualization of its load in the $N$-vector of loads.

The asperities in the temporary list are processed one 'by one by the upward-downward load distribution routines; once an asperitiy is processed it is dropped from the list. An avalanche of induced failures ends when no more asperities are left in the list, and the number of asperities processed gives directly the size of the burst.

The Monte Carlo algorithm has been tested with different random number generators (Press et al., 1986), and the output checked against known results. Figure 2 shows one of these checks, where a comparison

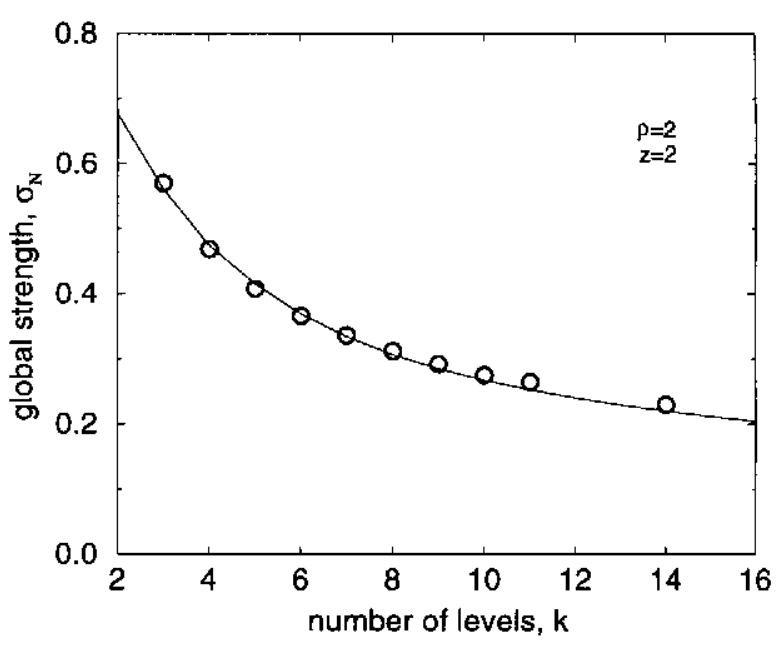

Fig. 2. Comparison of exact RG results (solid line) and Monte Carlo data (circles) for the global strength of $z=2$ fractal trees with $k=1, . .14$ levels.

is made between the Monte Carlo results (open circles) and the exact renormalization-group results (Newman and Gabrielov, 1991) (solid line) for the global strength of a fractal tree with coordination $z=2$. A Weibull probability distribution with $\rho=2$ is assumed for the strength of the individual asperities. The Monte Carlo data span the range $N=4-16384$ asperities, and inside this range the match with the exact $R G$ results is within statistical error (size of circles). Monte Carlo data are averages of $10^{5}$ to $10^{2}$ realizations for small and big trees, respectively. Because the fluctuations in global strength between realizations decrease markedly with system size, this results in a more-or-less constant statistical error in the whole range.

The main interest in this paper is concentrated on the size-frequency statistics of the partial bursts preceding the total collapse of the tree. Apart from these partial bursts, each Monte Carlo realization ends with a big burst where all the remaining asperities break; so, there is a gap in burst sizes between the partial bursts and the final, big one. Partial bursts follow a gross power-law behaviour and the results presented here concentrate on the fine structure of this relation for partial bursts.

Figure 3 is a normalized size-frequency plot for the bursts in $z=2$ fractal trees with sizes $N=1024$ (thick lines) and $N=16384$ (thin lines). Strengths for the asperities are drawn from a Weibull distribution with $\rho=4$. Solid lines are for the differential frequency count and dashed lines for the accumulated frequency count. Superimposed onto the general power-law trend we can see local maxima (differential curve) or local ramps (accumulated curve) periodic in the logarithm of the burst size. Local maxima appear at burst sizes of $2,4,8,16$, 32 , and 64 broken asperities, which seems to be related to the basic coordination of the fractal tree. 


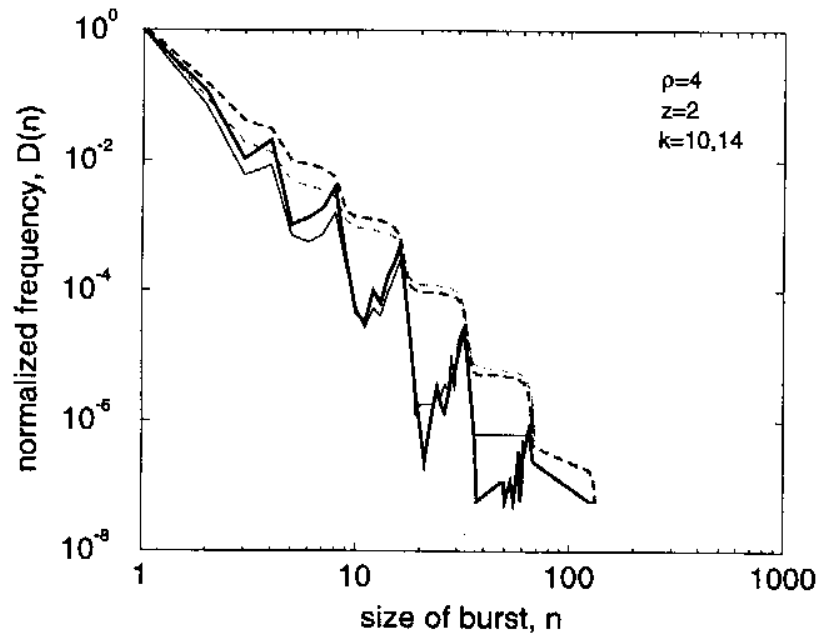

Fig. 3. Normalized frequency of bursts for fractal trees with $z=2, \rho=2$, and $k=10$ (thick lines) and $k=14$ (thin lines). Solid lines represent the differential frequency count and dashed lines the accumulated frequency count.

To confirm this last point, Fig. 4 plots the results for $N=1024$ (thick lines) and $N=16384$ (thin lines) trees with coordination $z=4$. Again, superimposed onto the gross power-law behaviour we observe log-periodic modulations with local maxima at burst sizes of 4,16 , and 64 broken asperities, which match the basic coordination $4^{n},(n=1,2,3)$ of the underlying tree structure.

\section{Conclusions}

As seen in Sect. 2, the size-frequency relation emerging from breaking bursts produced in large hierarchically organized sets of elements shows a gross power-law behaviour superimposed by a wavy pattern closely related to the coordination number $z$ used in the load-transfer scheme. To asses the credibility of a model in seismology, the first test to fulfill is the Gutenberg-Richter law, Eq. 2; therefore, these wavy patterns constitute a serious problem for this type of model.

When dealing with a fractal tree of very large $z$, the breaking process would proceed through the failure of large ELS sets and, accordingly, the final size-frequency relation would in fact coincide with that of the ELS model, Eq. 1. [In our simulations, we have noticed that for the same $\rho$ value, the slope of the log-log sizefrequency gross straight lines tends to increase as $z$ grows. Using the above mentioned qualitative argument, this slope would increase up to the mean-field limit $5 / 2$ of the ELS case.] But for ordinary small $z$ values the conspicuous appearance of the oscillations is unavoidable. Remember that $z=4$ was the value originally chosen to describe the stick-slip process in faults (Smalley et al., 1985).

It is important to recall that in this paper we have

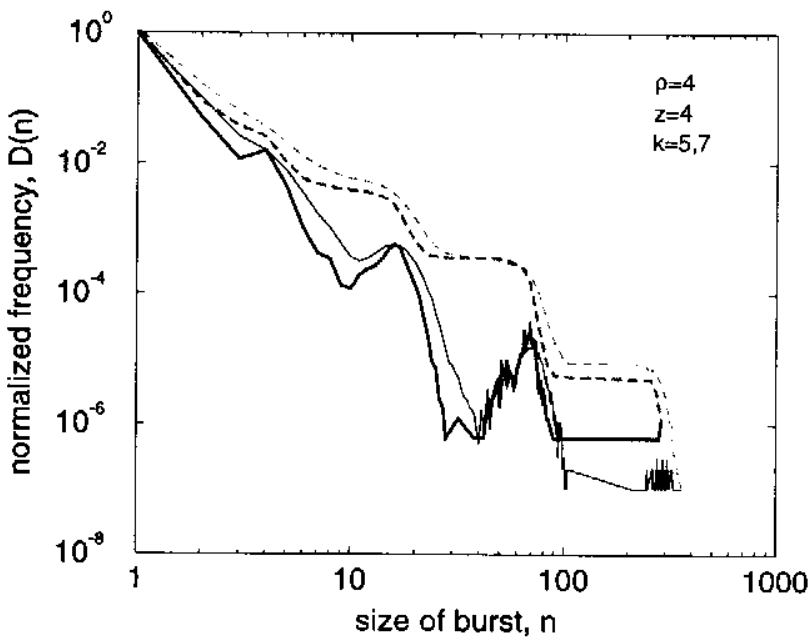

Fig. 4. Normalized frequency of bursts for fractal trees with $z=4, \rho=4$, and $k=5$ (thick lines) and $k=7$ (thin lines). Solid and dotted lines as for Fig. 3 .

used the static version of the model and hence, by definition, we are unable to discuss the time sequence of events leading to the total failure of the system. In this respect, one should be aware that this model, in the time-dependent version, has recently been studied by Newman et al. (1995) with this purpose. Their specific aim was to find out if the chain of partial failure events preceding the total failure resemble a logperiodic sequence. This was motivated by the amazing fit of this type obtained by Sornette and Sammis (1995) to data of the cumulative Benioff strain released in magnitude $>5$ earthquakes in the San Francisco Bay area before the October 17, 1989 Loma Prieta earthquake. Sornette and Sammis (1995) and Saleur et al. (1996) show that a power-law time-to-failure function can be derived from renormalization-group ideas where the main shock is viewed as a critical point. Furthermore, introducing first-order corrections to scaling, for the case of a discrete scale invariant system, the corrections take the form of a periodic function of the logarithm of the time to failure which modulates the zeroorder power-law. The analysis of Newman et al. (1995) is an attempt to implement these ideas using a specific well-defined model.

Another difference to bear in mind between our results and those of Newman et al. (1995) is that they analyse the time sequence of events occurring when individual large trees break, i.e., there is no average; our results, on the other hand, are obtained after an averaging process of many individual realizations.

Acknowledgements. This work was supported in part by the Spanish DGICYT (grant. PB93-0304). 


\section{References}

Christensen, K. and Olami, Z., Scaling, phase transitions, and nonuniversality in a self-organized critical cellular-automaton model, Phys. Rev., A46, 1829-1838, 1992.

Coleman, B. D., Time dependence of mechanical breakdown in bundles of fibres I: constant total load, J. Appl. Phys., 28, 1058-1064, 1957.

Daniels, H. E., The statistical theory of the strength of bundles of threads, I, Proc. R. Soc. London A183, 404-435, 1945.

Gómez, J. B., Iñiguez, D., and Pacheco, A. F., Size-frequency relation of earthquakes in load-transfer models of fracture, Nonlin. Procces. Geophys., 2, 131-135, 1994.

Gutenberg, B. and Richter, C. F., Frequency of earthquakes in California, Bull. seism. Soc. Am., 34, 185-188, 1944.

Hemmer, P. C. and Hansen, A., The distribution of simultaneous fiber failures in fiber bundles, J. Appl. Mech., 59, 904-909, 1992.

Jones, L. M. and Molnar P., Some characteristics of foreshocks and their possible relation to earthquake prediction and premonitory slip on fauls, J. Geophys. Res., 84, 3596-3608, 1979.

Kanamori, H., The nature of seismicity patterns before large carthquakes, in Simpson, D. W. and Richards, P. G. (eds), Earthquake prediction: an international review, Maurice Ewing Ser., 4, 1-19, A. G. U, 1981.

Kanamori, H. and Boschi, E. (Editors), Earthquakes, observation, theory and interpretation, North Holland, Amsterdam, 1983.

Lomnitz-Adler, J., Asperity models and characteristic earthquakes, Geophys. J. R. Astr. Soc., 83, 435-450, 1985.

Newman, W. I. and Gabriclov, A. M., Failure of hierarchical dis- tributions of fiber bundles, Int. J. Fracture., 50, 1-14, 1991.

Newman, W. I., Gabrielov, A. M., Durand, T. A., Phoenix, S. L., and Turcotte, D. L., An exact renormalization model for earthquakes and material failure. Statics and dynamics, Physica, D $77,200-216,1994$.

Newman, W. I., Turcotte, D. L., and Gabrielov, A. M., Logperiodic behaviour of a hierarchical failure modeI with application to precursory seismic activitation, Phy. Rev., E52, 48274835, 1995.

Phoenix, S. L., Stochastic strength and fatigue of fibers bundles, Int. J. Fracture, 14, 327-344, 1978.

Press, W. H., Flannery, B. P., Teukolsky, S. A., and Vetterling, W. T., Numerical Recipes: the Art of Scientific Computing, Cambridge Univ. Press, Cambridge, 1996.

Saleur, H., Sammins, C. G., and Sornette, D., Discrete invariance, complex fractal dimensions, and log-periodic fluctuations in seismicity, J. Geophys. Res., 101, 17661-17672, 1996.

Smalley, R. F., Turcotte, D. L., and Solla, S. A., A renormalization group approach to the stick-slip behaviour of faults, $J$. Geophys. Res., 90, 1894-1900, 1985.

Sornette, D., Elasticity and failure of a set of elements loaded in parallel, J. Phys., A22, L243-L250, 1989.

Sornette, D. and Sammis, C. G., Complex critical exponents from renormalization group theory of earthquakes: implications for earthquake prediction, J. Phys. I France, 5, 607-619, 1995.

Turcotte, D. L., Smalley, R. F., and Solla, S. A., Collapse of loaded fractal trees, Nature, $313,671-672,1985$.

Zhang, S. D. and Ding, E. J., Burst-size distribution in fiberbundles with local-sharing, Phys. Lett., A193, 425-430, 1994. 